\title{
CONTROL DEPLOYMENT OF MOBILE UNITS OF LARGE-SIZED SPACECRAFT
}

\author{
Fedor Mitin, Alexey Krivushov
}
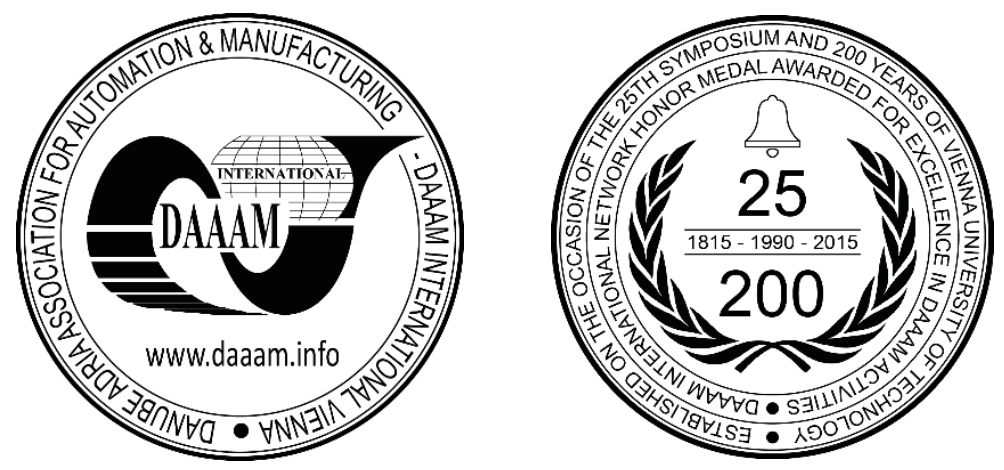

This Publication has to be referred as: Mitin, F[edor] \& Krivushov, A[lexey] (2017). Control Deployment of Mobile Units of Large-Sized Spacecraft, Proceedings of the 28th DAAAM International Symposium, pp.0773-0779, B. Katalinic (Ed.), Published by DAAAM International, ISBN 978-3-902734-11-2, ISSN 1726-9679, Vienna, Austria

DOI: $10.2507 / 28$ th.daaam.proceedings.109

\begin{abstract}
The variants of automatic deployment of mobile units of a large-sized spacecraft in orbit are considered. In view of the large-sized of the apparatus, its deployment from the folded state to the working one occurs in stages. The main task at each stage, in addition to reliable deployment, is to reduce the vibrations and deflections of the structure. At present, after the completion of the regular stage, it takes a considerable time to wait for the damping of the fluctuations and only then proceed to the next stage. This article explores possible options for deploying mobile units of a spacecraft: independent for each stage; joint for several stages, allowing to reduce fluctuations, deflections and, therefore, the time of opening the structure. Mathematical models of rotational and translational movements are presented. As an executive element, the application of an electric machine is considered, since it allows to control the deployment process. The results of numerical modeling allowing to evaluate the effectiveness of various variants of deployment are presented.
\end{abstract}

Keywords: large-sized spacecraft; deployment of a spacecraft; mathematical model

\section{Introduction}

At present, antennas are an integral part of modern spacecraft. The history of their creation is inextricably linked with the history of the development of space and rocket technology. Active research and development aimed at the creation in space of designs of various classes, having large size in one dimension or another, are introduced throughout the world.

Despite significant advances in the design of large-sized transformable space-based structures being deployment, the important task of their smooth and reliable deployment remains [1-5].

A distinctive feature of large-size spacecraft (large-size antennas, space telescopes, etc.) is the presence in their composition of a multitude of mobile units. Of particular interest are transformable antennas, the profile of which can be changed both at the stage of preparation for work and in the process of functioning. Due to the large size, the translation of the reflector from the folded state to the workers is divided into stages that can be regarded as independent of each other.

Deployment of various types of large-size reflectors (trusses, umbrellas, rings, etc.) is done at the expense of the energy of springs or deformed elastic elements [6-8]. 
The disadvantages of this method of deployment include the inability to control the deployment process, the presence of fluctuations and deflections of the structure after completion of the deployment stage, the need to create a layout and perform additional calculations to determine the degree of compression of springs or twisting of elements.

An alternative to classical systems of deployment can be automatic systems in which the energy for the deployment of reflector elements is produced by drives, in particular an electric machine. The application of this type of actuator will allow to control the process of opening, to reduce the fluctuations and deflections of the design of a large-sized spacecraft.

\section{Mathematical model of mobile units}

The process of deployment of a large-sized spacecraft can be divide into several separate stages. Let us consider in more detail the most frequently encountered types of motion describing these stages: rotational and translational.

In the course of the study, it was proposed to simultaneously open several elements of a large-sized spacecraft in order to shorten the time taken for the apparatus to move to the working position. Consider the problem of opening the root element and the simultaneous extension of the intermediate element of the end elements of the power frame, shown in Figure 1.

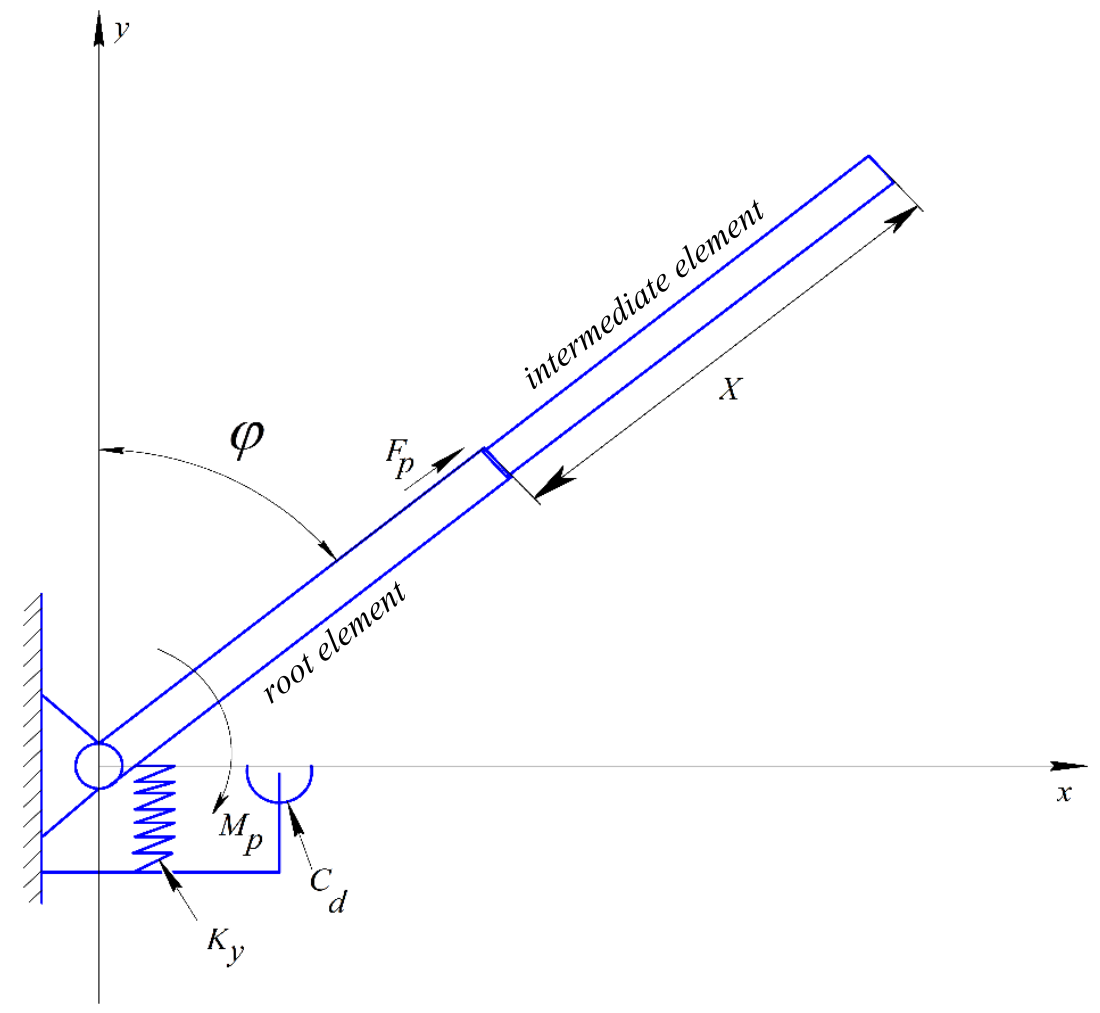

Fig. 1. Scheme of mutual arrangement of the elements of the base of the power frame

The system describing the joint deployment is a combination of two systems: angular motion and translational motion, the mathematical models of which are presented in $[9,10]$. We obtain the system:

$$
\begin{aligned}
& \dot{\varphi}=w-h, \\
& \dot{w}=\frac{M_{p}-M_{t r}-\left(k_{y} \cdot \Delta \varphi+C_{d} \cdot \dot{\varphi}\right) \cdot H(\Delta \varphi)-\mu \cdot \dot{\varphi}}{I}, \\
& \dot{x}=V \pm \Delta x_{s z}, \\
& \dot{V}=\frac{F_{p}-F_{t r}-\left(k_{y} \cdot \Delta x+C_{d} \cdot \dot{x}\right) \cdot H(\Delta x)-\mu \cdot \dot{x}}{m} ;
\end{aligned}
$$

where $\varphi$ is the angle of rotation of the root element; $\omega$ is the angular velocity of rotation; $l$ is the length of the rotating part; $\rho$ is the material density; $E$ is the Young's modulus for the material; $I$ is the moment of inertia; $k_{y}$ is coefficient of rigidity of the elastic component of the stop (lock) model; $C_{d}$ is the coefficient of damping of the viscous component of the stopping device; $\Delta \varphi=\left(\varphi-\varphi_{\mathrm{K}}\right)$ is the relative angle corresponding to the moment of installation at the stop (lock); $\mu$ is the coefficient of dissipativity; $H(\Delta \varphi)$ is a function that takes the value $0(\Delta \varphi>0)$ or $1(\Delta \varphi<0)$; $M$ is the engine torque; $M_{t r}=\varphi_{i s h} \varphi_{r s} M_{r r}+M_{s l}+M_{\text {seal }}+M_{\text {drag }}, M_{r r}$ is the rolling friction moment; $M_{s l}$ is the sliding friction moment; $M_{\text {seal }}$ 
is the friction moment of the seals; $M_{\text {drag }}$ is the friction moment due to the resistance of lubrication, agitation, sprays, etc., $\varphi_{i s h}$ is the reduction factor due to the heating of the lubricant; $\varphi_{r s}$ is the reduction factor in the kinematic fasting mode; $x$ ist he displacement; $V$ is the linear velocity; $\Delta x_{s z}=\frac{F_{p} \cdot l}{E \cdot S} ; F_{p}=k_{f} \cdot M_{p}$ is the useful force, push intermediate element; $F_{t r}=$ $\mu_{t r} \cdot N_{i}$ is the frictional force; $\mu_{t r}$ is the coefficient of sliding friction of the guide sections; $N_{i}$ is the normal forces in the sliding supports of the $i$-th section; $\Delta x=\left(x-x_{\mathrm{K}}\right)$ is the relative movement corresponding to the moment of installation at the stop (lock); $H(\Delta x)$ is a function that takes the value $0(\Delta x>0)$ or $1(\Delta x<0)$.

During the operation of the system, it may be necessary to take into account the bending of the loose end of the element. Starting from [11] we consider the applicability of the approximate theory. The approximate equation for calculating the deflection value is calculated as:

$$
h=\frac{M(l+\Delta l)}{2 E I}
$$

where $\Delta l$ is the elongation of the element; $M=M_{p}-M_{t r}-\left(k_{y} \cdot \Delta \varphi+C_{d} \cdot \dot{\varphi}\right) \cdot H(\Delta \varphi)-\mu \cdot \dot{\varphi}$ is the total moment acting on the base of the power frame.

To create a useful torque $M_{p}$, we choose an electric motor that is most suitable for the problem, that is, Taking into account the field of application, environmental conditions, service life, power source, operation cycle, heat generation, efficiency, controllability, torque, speed, weight. Analyzing current trends in the application of electric motors for similar purposes [12-14], one can come to the conclusion that the most suitable are brushless (gate) DC motors. Since they have a long service life, they provide high moments, high efficiency and low heat dissipation.

The torque is calculated by the equation [14]

$$
\mathrm{M}_{p}=\frac{m p E_{0} U_{1}}{w_{1} X_{c}} \operatorname{sin\vartheta }
$$

where $m$ is the number of phases of the rotor; $p$ is the number of pairs of poles of the magnetic field, $E_{0}$ is the effective value of the emf on the stator winding; $U_{l}$ is the supply voltage, $x_{c}$ is the synchronous resistance $\left(x_{c}=x_{\sigma}+x_{a}\right.$, where $x_{\sigma}$ and $x_{\mathrm{a}}$ are the inductive scattering resistances and reactions respectively), $\vartheta$ is the angle of error (between $U_{l}$ and $E_{0}$, for the motor is within $[0, \pi / 2])$.

\section{Modeling}

The first method of joint deployment of the stages is the use of the engine for each of the components of the motion. In modeling the dynamics of the joint opening of the reflector, the damping factors and the elastic component of the stop varied, the actuators for both types of movements had the same parameters and were switched on simultaneously. The following system parameters were selected during simulation: ABS material plastic QHF 0140: $\rho=1040 \mathrm{~kg} \cdot \mathrm{m}^{3}, E=$ $9900 \cdot 10^{6} \mathrm{~Pa}, U_{l}=5 \mathrm{~V}$, retractable part with length $x_{k}=2 \mathrm{~m}$, angular movement stop $\varphi_{k}=1 \mathrm{rad}$. The behavior of the system is shown in Figures 2-3.

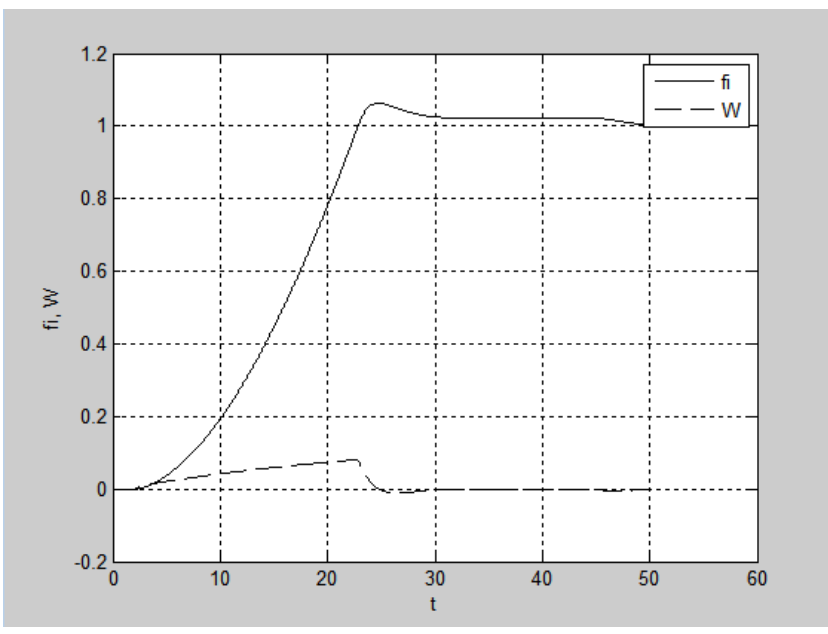

a)

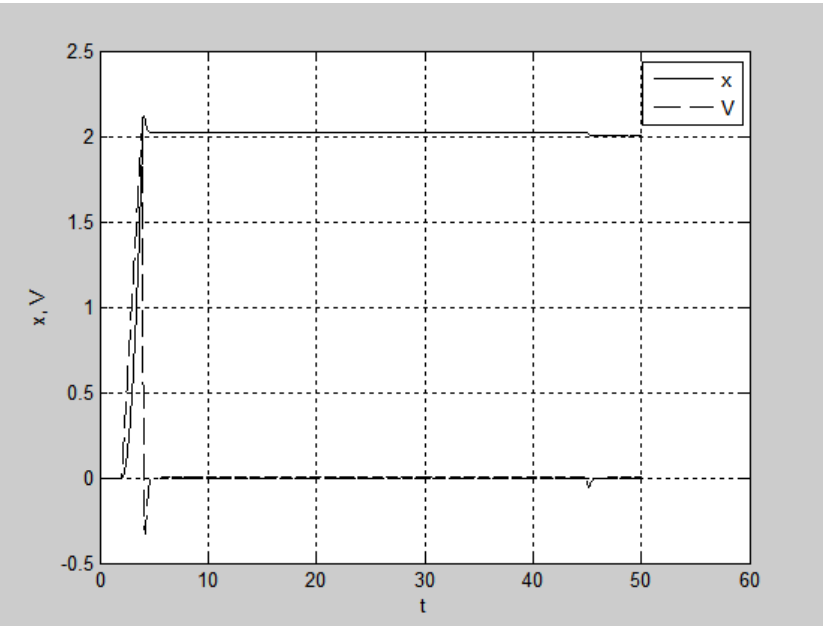

b) 


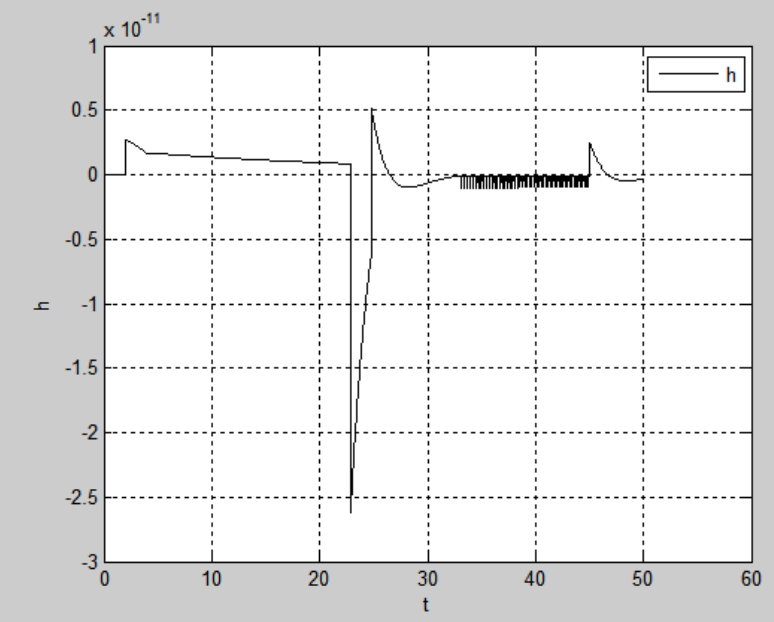

c)

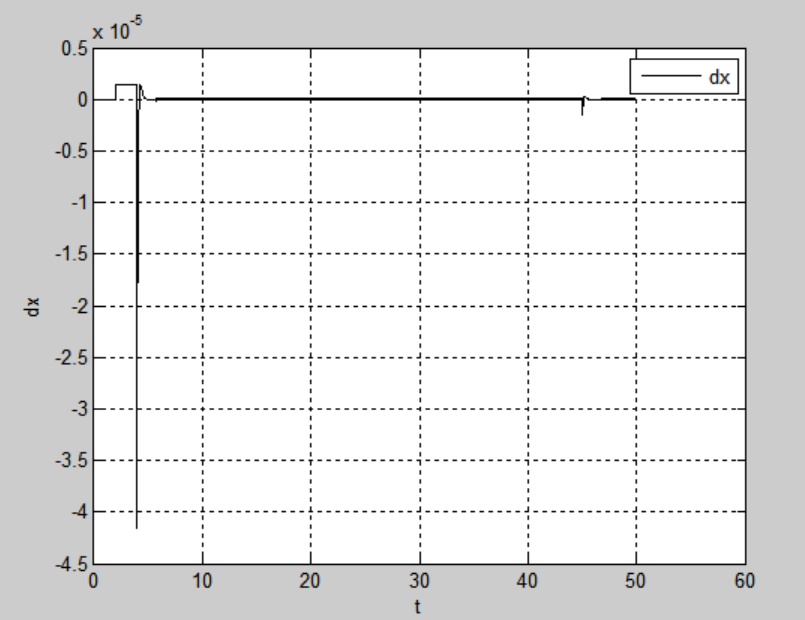

d)

Fig. 2. The characteristic of the dynamics of joint deploymentof the elements: a) the graph of $\varphi(t)$ and $\omega(t)$ at $k_{y}=10$, $\left.C_{d}=25 ; \mathrm{b}\right)$ the graph of $x(t)$ и $V(t)$ at $k_{y}=100, C_{d}=25$; c) the graph of $h(t)$; d) the graph of $\Delta \boldsymbol{x}_{\boldsymbol{s z}}(t)$

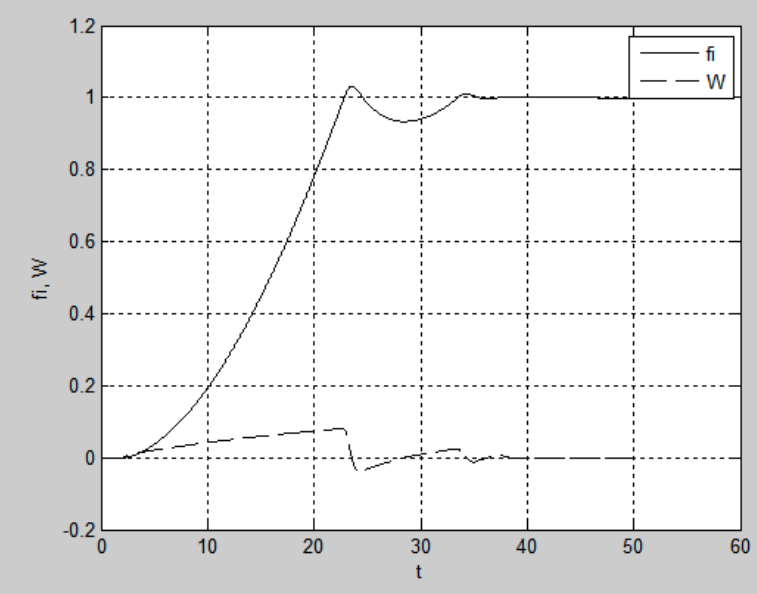

a)

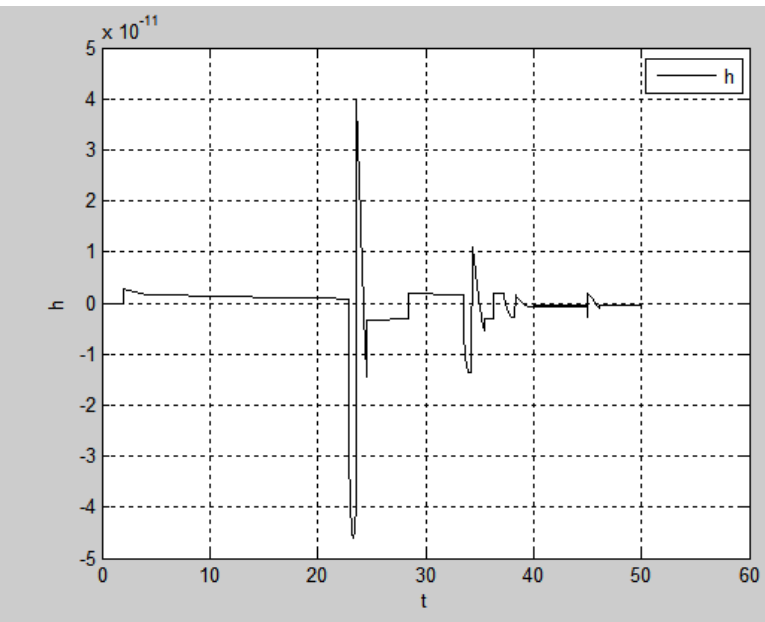

c)

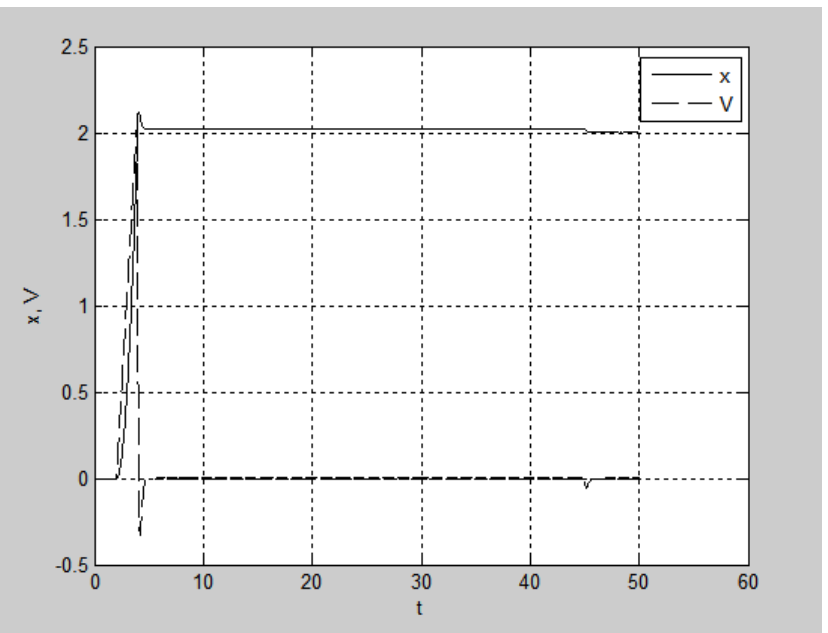

b)

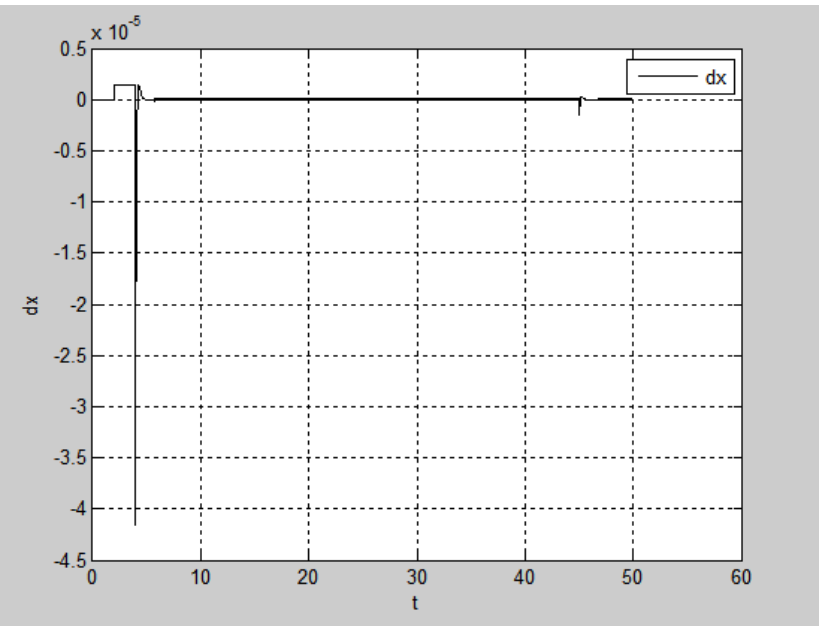

d)

Fig. 3. The characteristic of the dynamics of joint deploymentof the elements: a) ) the graph of $\varphi(t)$ and $\omega(t)$ at $k_{y}=100$, $C_{d}=25$; b) the graph of $x(t)$ и $V(t)$ at $k_{y}=100, C_{d}=25$; c) the graph of $h(t)$; d) the graph of $\Delta \boldsymbol{x}_{\boldsymbol{s z}}(t)$ 
Based on the above, we can make the following conclusions. The use of several executive devices allows implementing various programs for the deployment of design elements. The disadvantages include the fact that with the increase in the number of executive machines, the mass of the entire design, the inertia of the elements in the design, will also grow.

The second way is to use centrifugal force to deployment intermediate element. In this method, the useful force $F_{p}$ is created by the centrifugal force. The system describing the joint expansion of the structural elements has the same form as the system $(1-4)$, except that $F_{p}=0,5 \cdot m \cdot \omega^{2} \cdot(x+0.5 \cdot l)$.

During the simulation, the same system parameters were used as for the first method. From figure 4 it can be seen that the actuator of the angular motion creates insufficient centrifugal force for the full extension of the intermediate element at the initial parameters. Based on this, the control signal $U_{l}$ was increased from $5 \mathrm{~V}$ to $15 \mathrm{~V}$. Due to the increase in the control signal, the torque also increased. Figure 5 shows the process of expansion and extension at $U_{l}=15 \mathrm{~V}$. From the obtained characteristic, it can be concluded that it was possible to achieve setting on the stops of both the root element and the intermediate element of the reflector.

This embodiment is devoid of the disadvantage of the first method in the form of additional weight of the actuators. The problem of voltage control is complicated, since it becomes necessary to vary $U_{l}$ the actuator based on the desired characteristics of not only the angular motion but also the translational component.

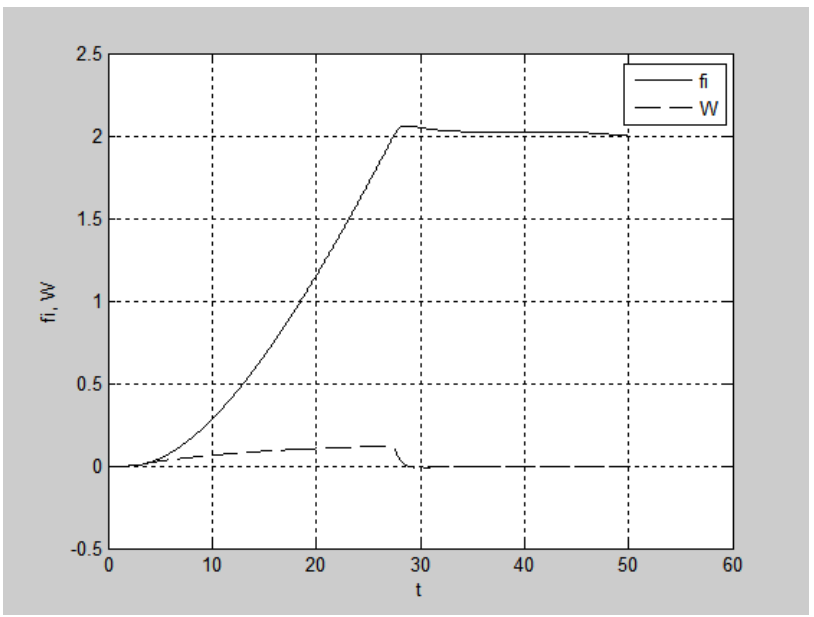

a)

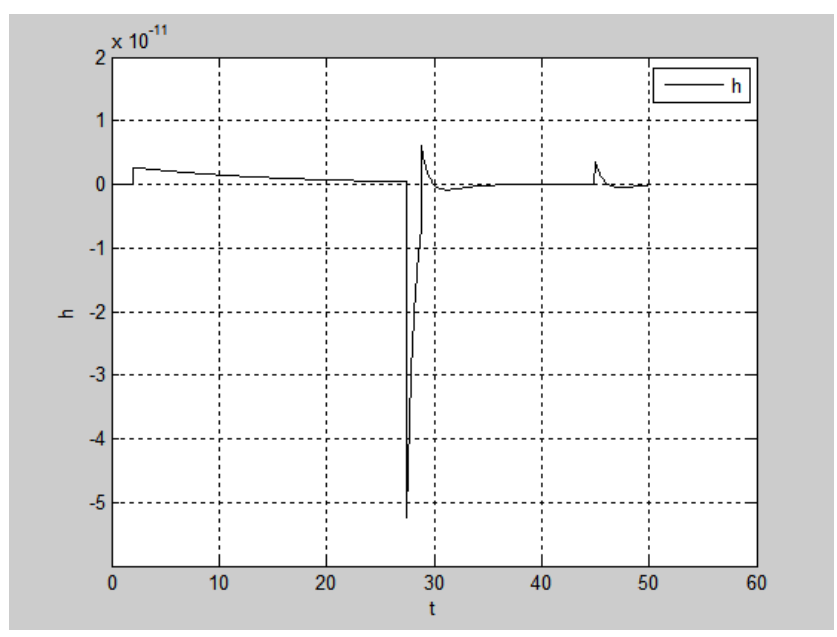

c)

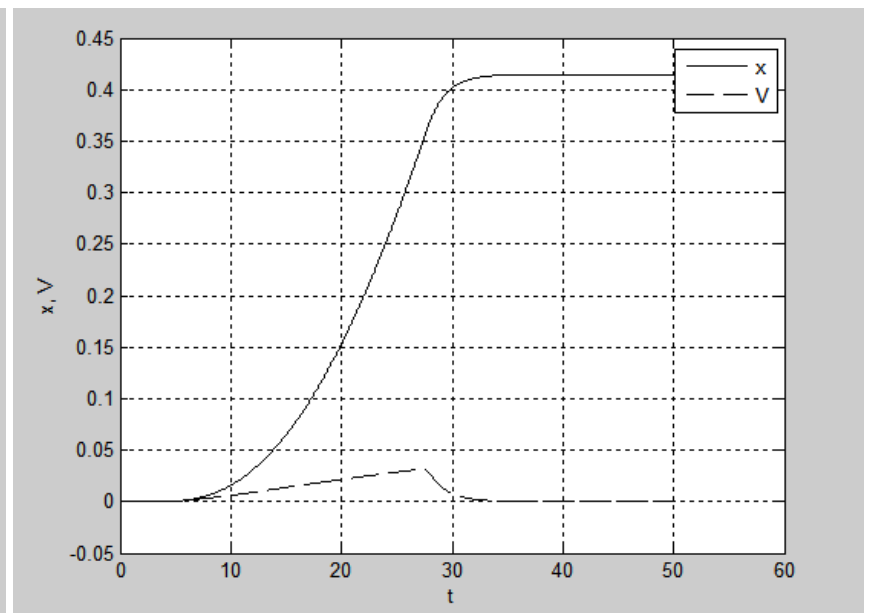

b)

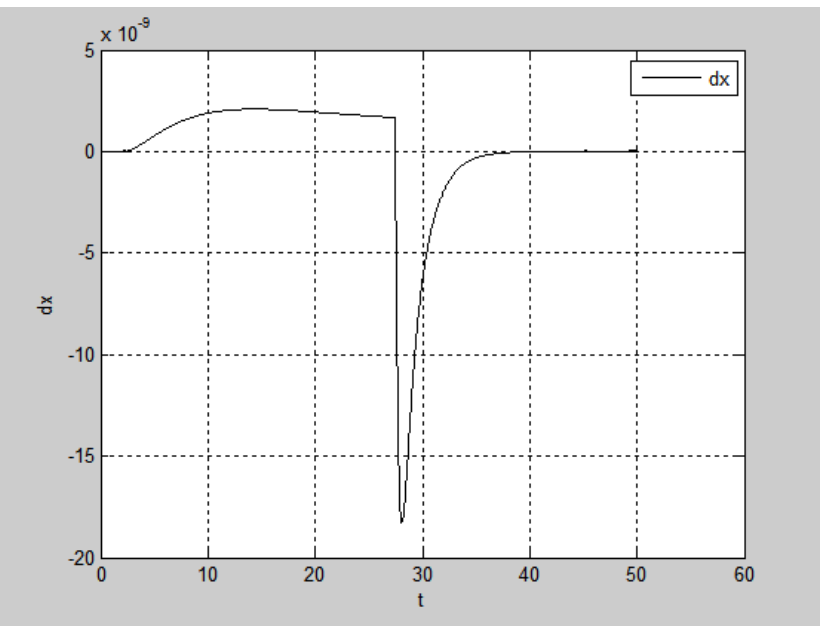

d)

Fig. 4. The characteristic of the dynamics of joint deployment of the elements: a) the graph of $\varphi(t)$ and $\omega(t)$ at $U_{l}=5 \mathrm{~V}$, $\left.k_{y}=10, C_{d}=25 ; \mathrm{b}\right)$ the graph of $x(t)$ and $V(t)$ at $\left.k_{y}=100, C_{d}=25 ; \mathrm{c}\right)$ the graph of $h(t)$; d) the graph of $\Delta \boldsymbol{x}_{\boldsymbol{s z}}(t)$ 


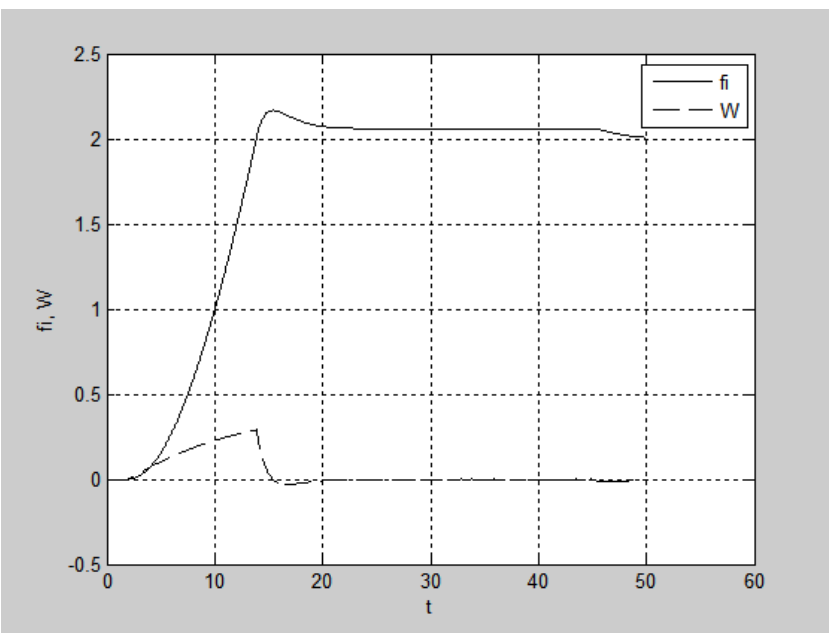

a)

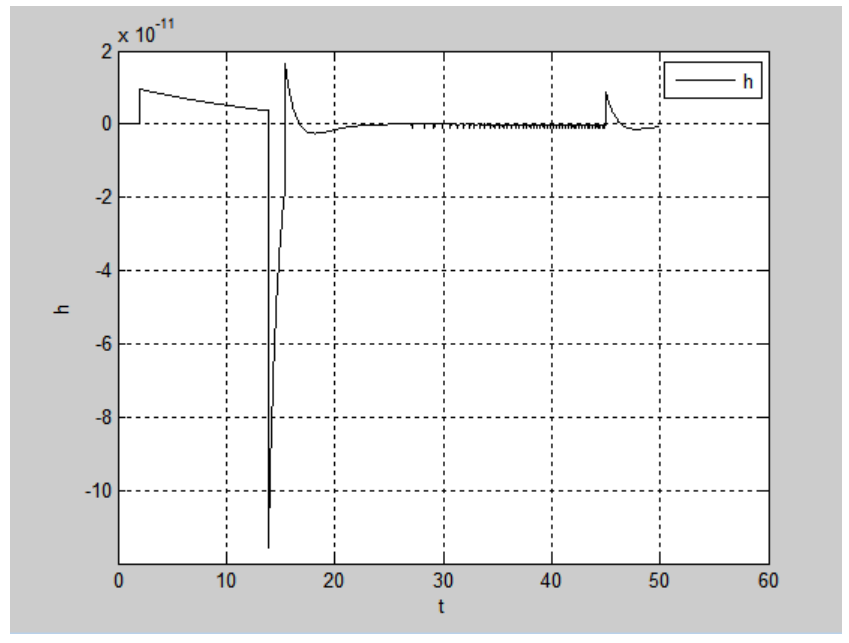

c)

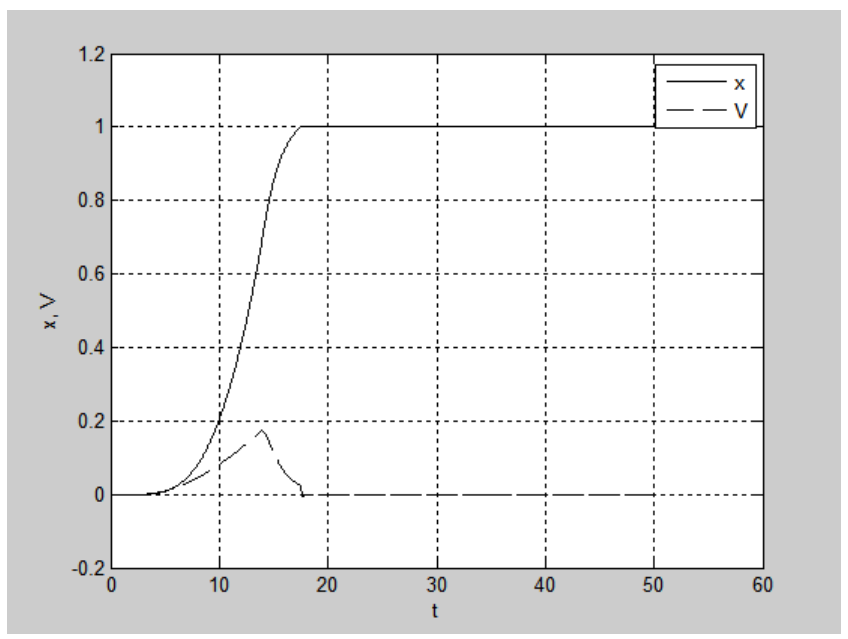

b)

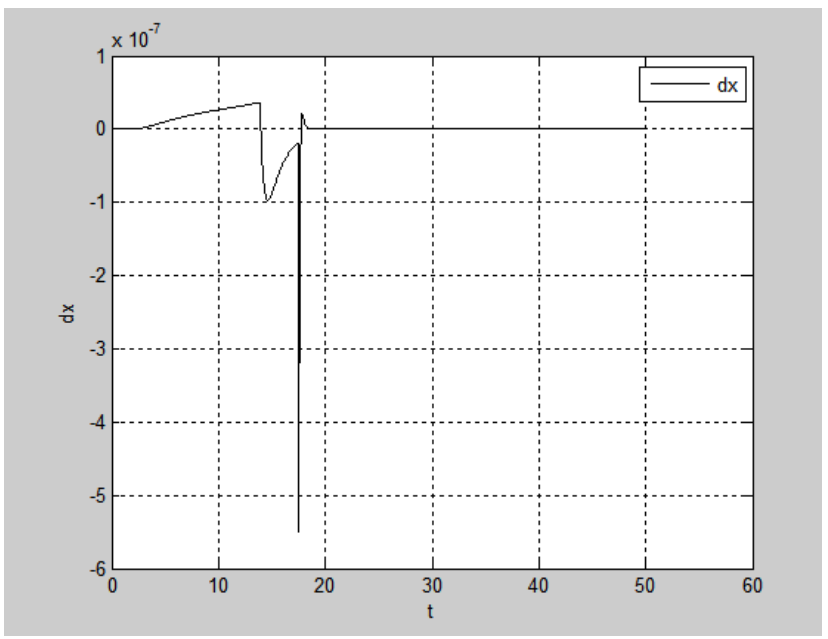

d)

Fig. 5. The characteristic of the dynamics of joint deploymentof the elements: a) the graph of $\varphi(t)$ and $\omega(t)$ at $U_{l}=15 \mathrm{~V}$, $\left.k_{y}=10, C_{d}=25 ; \sigma\right)$ the graph of $x(t)$ and $V(t)$ at $\left.k_{y}=100, C_{d}=25 ; \mathrm{c}\right)$ the graph of $\left.h(t) ; \mathrm{d}\right)$ the graph of $\Delta \boldsymbol{x}_{\boldsymbol{s z}}(t)$

\section{Conclusion}

In the course of the study, two universal models of simultaneous realization of several stages of the reflector opening were obtained. Versatility lies in the possibility of using them for structures with different parameters: from changing the material, mass, dimensions to selecting the control body (motor). Accounting for such important parameters as the magnitude of the bending of the structural elements, dissipation, dynamic elongation, the influence of the properties of the stop on the angular motion of the body when framed on the stop, made it possible to describe the behavior of the system with a sufficiently high accuracy.

The main interest in choosing the control option was to find a version of the deployment, which reduces the vibrations and deflections of the structure. The two options considered allow us to manage the deployment process, which will allow us to further implement the control algorithm solving this problem. In order to implement the first variant, an additional actuator is required, the second variant is deprived of this drawback and is preferred. When using both methods, it is necessary to take into account the limitations on maximum loads and design moments, since the forces created by the system can exceed critical forces.

The results of these studies can be used to select the executive body, assess the necessary efforts. This mathematical model is approximate. To improve the quality of the system and the accuracy of the results, it is necessary to consider options for solving the direct and inverse problems of dynamics for physical systems, including the account of the energy components of the motion presented in $[15,16]$.

In the future, to improve the characteristics of the deployment of the reflector, it is supposed to solve the problem of a smooth approach of the structural elements to the stops and setting them. To solve this problem, it is proposed to use the methods of optimal control. 


\section{Acknowledgments}

The research was carried out within the framework of the implementation of the Federal Target Program "Research and Development in Priority Areas for the Development of the Russian Science and Technology Complex for 20142020", the Ministry of Education of Russia, agreement No. 14.577.21.0201 (unique identifier RFMEFI 57715X0201): "Creating a highly efficient wireless system Directional transmission of energy and information for managing the state of space-based objects based on laser and fiber-optic technologies. " The work was carried out on the equipment of the Center for Innovative Technologies, the Center for Additive Technologies and Volume Scan, the Center for RadioTechnical and Optical Measurements, the Center for IPI Technologies, the Center for Materials Research, the Center for Educational Research Center of expert testing and quality control of new functional materials of innovative and nanotechnology. "

\section{References}

[1] Bei N.A., Zimin V.N. (2005). Transformable antennas of large dimensions for geostationary spacecraft, Antennas, No. 10(101), 2005, pp. 24-27.

[2] Lopatin A.V., Rutkovskaya M.A. (200)7. Overview of the constructions of modern transformed space antennas (Part 1), Vestnik of Siberian State University of Automation, No. 2, 2007, pp. 51-57.

[3] Seefeldt Patric, Spietz Peter, Sproewitz Tom, Grundmann Jan Thimo, Hillebrandt Martin, Hobbie Catherin, Ruffer Michael, Straubel Marco, Three Norbert, Zander, Martin. (2017). Gossamer-1: Mission concept and technology for a controlled deployment of gossamer spacecraft, Advances in Space Research, Vol. 59, No. 1, 2017, pp. 434-456.

[4] Khoroshilov, V. S., Zakrzhevskii, A. E. (2012). Dynamics of spacecraft due to elastic ring antenna deployment, Vibrations in Physical Systems, Vol. 25, 2012, pp. 229-234.

[5] Isaenko, Sergei; Sochivko Oleg \& Dalyaev Igor. (2016). Analysis of Vibration Modelling Validity of Space-Borne Robotic System, Proceedings of the 26th DAAAM International Symposium, ISSN 1726-9679, Vienna, Austria, 2016, pp. 0548-0553

[6] Grinevich D.V. (2013). Investigation of the dynamics of unfolding extended structures, Questions of electromechanics, Proceedings of VNIIEM, Vol. 134, 2013, pp. 37-42.

[7] Kabdulin G.V., Komkov V.A., Melnikov V.M., Harlov B.N. (2009). Dynamics of controlled opening by centrifugal forces of space structures with compensation of the kinetic moment, Journal of Cosmonautics and Rocket Science, No. 1 (54), 2009, pp. 189-198.

[8] Bagheri Ghaleh, P. and Malaek, S.M. (2015). On dynamic stiffness of spacecraft flexible appendages in deployment phase, Aerospace Science and Technology, Vol. 47, 2015, pp. 1-9.

[9] Krivushov A.I. (2017). Creation and investigation of the mathematical model of the rotational motion of the spokes of a large-sized space-based reflector, Questions of radio electronics, No. 7, 2017, pp. 122-126.

[10] Mitin F.V. (2017). Development and analysis of the mathematical model of translational motion of transformed space structures, Questions of radio electronics, No. 7, 2017, pp. 117-121.

[11] Rabotonov Yu. N. (1962). Resistance of materials, Fizmatgiz, Moscow, 1962.

[12] Woldek A.I., Popov V.V. (2007). Electric cars. Introduction to electromechanics. DC machines and transformers: A textbook for high schools, Peter, St. Petersburg, 2007.

[13] Ivashov E.N., Panfilova E.S., Shikhov A.I. (2014). The choice of the electric motor for vacuum switching equipment, Advances in modern natural science, No. 12 (Part 4), 2014, pp. 409-411.

[14] Usoltsev A.A. (2009). General electrical engineering: Textbook, St. Petersburg State University ITMO, St. Petersburg, 2009.

[15] Kostin G.V., Saurin V.V. (2009). The method of integro-differential relations for the analysis of natural oscillations of membranes, PMM, Vol. 73. No. 3, 2009, pp. 459-473.

[16] Kostin G.V., Saurin V.V. (2010). Modeling of spatial motions of an elastic beam on the basis of the method of integro-differential ratios, Modern problems of continuum mechanics, Proceedings of the XIV International Conference, Vol. 2, Rostov - on - Don, Azov, 2010. pp. 165-169. 